\title{
Verändert Covid-19 unsere Konzeption von Spiritual Care? Von Michel Foucault inspirierte Thesen unter dem Eindruck der unbewältigten Corona-Krise
}

\author{
Eckhard Frick SJ
}

Bedrängnis bewirke Geduld (bypomonē), schreibt Paulus (Röm 5,3). Eine derartige hypomonē („Darunterbleiben“) wird gegenwärtig von den Menschen in Europa verlangt: In einer kollektiven Geduldsprobe bisher ungekannten Ausmaßes sollen sie zeigen, dass sich Bürgerinnen und Bürger freiheitlicher Demokratien ähnlich konsequent dem „Shutdown“ unterwerfen wie in Diktaturen, die schon vor der Corona-Krise einige Routine im Überwachen und Strafen (Foucault 1975/1994) hatten (Hannah 2020). Sie sollen zeigen, dass sie als Unterworfene (sub-iectil-ae), als Integral unter der epidemiologischen Kurve der Johns Hopkins University ausharren können, deren Verlauf sich nach Möglichkeit abflachen und verlängern soll. In diesem Beitrag werden Thesen zu Spiritual Care in der Situation des Shutdown formuliert - unter dem Vorbehalt der noch nicht abgeschlossenen und folglich noch nicht abschließend beurteilbaren Krise (ausführlicher in: Frick 2020a).

\section{Welches Modell von Spiritual Care ist diskursfähig?}

Der Begriff „Spiritual Care" ist vieldeutig und kann verwendet werden als Synonym für kirchliche Seelsorge, als generische, interreligiöse Bezeichnung für die Seelsorgen verschiedener Religionsgemeinschaften sowie für gemeinsame spirituelle Sorge der Gesundheitsberufe (Frick/Roser 2011). Diese gemeinsame Sorge kann entweder im ausschließenden Gegensatz zur kirchlichen Seelsorge (Karle 2010) oder im Rabmen eines inklusiven Modells (Frick 2020b; Peng-Keller 2020) konzipiert werden. 


\section{Ist die Corona-Krise relevant für den Diskurs?}

Die Covid-19-Pandemie ist ein medizinisches Ereignis, das gleichzeitig mit seiner Globalisierung zum "Shutdown" aller Gesellschaftsbereiche führt, die nicht als „systemrelevant" gelten. Kriterien für die Systemrelevanz ergeben sich aus dem dominierenden virologisch-epidemiologisch bestimmten Covid-19-Diskurs, dem sich alle gesellschaftlichen Bereiche, auch nicht-systemrelevante, unterordnen. Covid-19 bildet also einen eigenen, ausschließlichen und ausschließenden MegaDiskurs, der nach und nach ergänzt wird durch argumentatives Erstreiten von Ausnabmeregelungen (z.B. bei unmittelbar bevorstehendem Tod, der nicht, ,aufschiebbar" ist) und die Debatte über „Exit-Strategien“. Derartige Ausnahmen bestätigen die herrschende Diskurs-Regel, die sich selbst durch DiskursVerknappung sowie Ausschluss von Themen und Sprechenden stabilisiert.

Es ist inzwischen Routine, muss deshalb in Erinnerung gerufen werden: Covid-19, also ein medizinisches Thema, eine Entität genetischer Information, die ohne Wirt nicht lebensfähig ist, hat sich zur bleibenden Schlagzeile, zum dominierenden Diskurs-Initiator entwickelt. Andere, viel häufigere Krankheits- und Todesursachen, soziale Spannungen, Kriege, Flucht und Vertreibung, nicht zuletzt die Klimakrise: All dies tritt in den Hintergrund. Die Pandemie führt zum Covid-19-Monolog.

\section{Pastoralmacht der Politiker und Ärzte}

Foucault (1979/1981) prägt den Begriff „Pastoralmacht“, indem er das biblische Motiv der Sorge des guten Hirten säkularisiert und auf individualisierte Überwachung des Seelenheils, von Leib und Leben der "Schafe" anwendet. In der Medizin prägt das pastorale Prinzip die therapeutische Beziehung (Rose 2001) und den epidemiologischen sowie gesundheitspolizeilichen Datenaustausch (Cooper 2019).

Chassé-croisé nennt man im Französischen den gekreuzten Wechselschritt im Tanz, im übertragenen Sinn die Ablösung einer Gruppe durch ihre Nachfolger am selben Ort, z. B. die Übernahme von Hotelzimmern, die zuvor von den Abreisenden bewohnt wurden, durch ankommende Gäste. Ein derartiges chassé-croisé ist gegenwärtig zwischen Ärzten und Seelsorgenden zu beobachten: Die Ärzte sind (im Verein mit den Politikern) die neuen Hirten, die sich um das Seelenheil der Herde sorgen, während die bisherigen Hirten sich restlos dem medizinischen Modell unterordnen und allenfalls darum kämpfen, von der Pastoralmacht als „notwendig“ zu Pati- 
enten und Mitarbeitenden vorgelassen zu werden. Freilich ist der "Tanz“ noch nicht zu Ende. Nicht nur Afrika, sondern auch Europa braucht „Spiritual Brokers“ (Winiger 2020), die zwischen der biomedizinischen Sphäre und der spirituellen vermitteln.

\section{Biopolitik als Perfektionierung des Panoptismus}

Foucault (1975/1994) verknüpft das Panopticon (Bentham 1791) als Symbol der idealen Überwachung in Gefängnissen, Krankenhäusern, Schulen usw. mit Modellen der Seuchen-Bewältigung. Nicht mehr der einzelne Aufseher bewacht (mit Argusaugen) eine Vielzahl von Parzellen. Vielmehr perfektioniert und demokratisiert sich das „Auge des Gesetzes“ zu einer potenziell ständigen gegenseitigen Sichtbarkeit und Kontrolle (Synoptismus). Die molekulare Biopolitik (Rose 2007) mit ibrem Zugriff auf Big Data, Mobilitäts-Tracking und GenomKartierung bringt den Panoptismus zum Exzess alltäglicher Routine.

Foucault schildert die Überwachung in Seuchen-Zeiten: Durch Parzellierung werden alle in die eigenen Wohnungen eingeschlossen. „Herausgehen wird mit dem Tode bestraft. [...] Wer sich rührt, riskiert sein Leben: Ansteckung oder Bestrafung“ (Foucault 1975/1994, 251). Modellgebend sind die Pest und die Lepra: „Im Hintergrund der Disziplinierungsmodelle steht das Bild der Pest für alle Verwirrungen und Unordnungen, wie das Bild des Aussatzes hinter den Modellen der Ausschließung steht" (Foucault 1975/1994, 255).

Wir haben es also mit zwei entgegengesetzten Bildern von Disziplin zu tun: auf der einen Seite die Disziplin als Blockade, als geschlossene Anstalt, die innerhalb bestimmter Grenzen auf negierende Funktionen ausgerichtet ist: Bannung des Übels, Unterbrechung der Beziehungen, Aufhebung der Zeit. Auf der anderen Seite die Disziplin als panoptischer Betrieb, als Funktionszusammenhang, der die Ausübung der Macht verbessern, d.h. beschleunigen, erleichtern, effektiver machen soll: ein Entwurf subtiler Zwangsmittel für eine künftige Gesellschaft (Foucault 1975/1994, 269).

Näher an unserer Realität als das Pest/Lepra-Modell ist das Modell der Pockeninfektion (Sarasin 2020). Es basiert darauf,

[...] dass die Macht den Traum aufgibt, die Pathogene, die Eindringlinge, die Krankheitskeime vollständig auszumerzen, die Gesellschaft »in die Tiefe« hinein zu überwachen und die Bewegungen aller Indivi- 
duen zu disziplinieren. Die Macht koexistiert vielmehr mit dem pathogenen Eindringling, weiß um sein Vorkommen, sammelt Daten, erstellt Statistiken, lanciert »medizinische Feldzüge«, die durchaus den Charakter der Normierung und Disziplinierung der Individuen annehmen können - aber die Disziplin, gar die vollständige, kann in der Moderne kein vernünftiges Ziel der liberalen Macht mehr sein. Dort, wo sie dies dennoch anstrebt, wo die Macht vom Pocken-Modell zum Pest-Modell zurückkehren möchte, wird sie totalitär (Sarasin 2005, 103).

Die neue Form der Panoptizität ist die Herrschaft mit Zahlen und Daten (Cooper 2019), „Nexistenz in der Matrix“:

Die Existenz in der Matrix beinhaltet ein diffuses Gefühl der Existenz ohne Geheimnisse, das geradezu offensiv verleugnet werden muss. Die Beruhigungsmantras lauten: Wer soll denn die ganzen Daten überhaupt verarbeiten? Wer interessiert sich schon für meinen Blutdruck? Soll der Geheimdienst doch alle meine Telefonate speichern, es gibt sowieso nichts Wichtiges zu hören. Das Interessante an diesen Beruhigungsreden ist, dass gar nicht mehr in Abrede gestellt wird, dass man öffentlich existiert. Es wird hingenommen als etwas, das wohl keine Konsequenzen haben wird. Zugleich wird ein diffuses Gefühl des Beobachtet-werdens auf Dauer gestellt. Wir leben nicht in einem Panoptikum, wo von einer Zentralstelle aus mit bekannten Intentionen beobachtet wird, sondern wir leben in einem generalisierten Panoptikum, in dem von vielen Beobachtungspunkten aus mit schwer einschätzbaren Intentionen beobachtet wird. Und: ich selbst könnte, wenn ich die technischen Möglichkeiten hätte, zurückbeobachten. Im Verhältnis dazu ist das von Foucault zur Beschreibung der Disziplinarmacht herangezogene Panoptikum mit Zentralstelle ein überschaubarer und gemütlicher Ort. Das alte Panoptikum führt zur Normalisierung. Man verhält sich so, als würde man beobachtet, auch wenn man gar nicht beobachtet wird. Das Neuartige des generalisierten Panoptikums ist die Unbestimmtheit der vernetzten Normalitätserwartungen und die Unbestimmtheit der Konsequenzen. Paradoxerweise wird gerade das Recht auf informationelle Selbstbestimmung zu einer Steigerung der Beobachtbarkeit führen (Lindemann 2015/2019, 62).

Die aktuelle Corona-Krise zeichnet sich, wie eingangs erwähnt, durch Ungewissheits-(In-)Toleranz (Spitzer 2019) aus. Biopolitik wird weitgehend zur Affektpolitik, was sich auf das Verhältnis zur Norm (a), zum Zeitregime (b) und zur Natur (c) auswirkt (Bihl 2019): 
(a) Während im Pest/Lepra-Modell die Norm der Disziplin gewissermaBen einen idealen Richtwert darstellte, tritt an die Stelle der Normalisierung ein Prozess der Normfindung, der den tatsächlichen statistischen Verteilungen folgt, anstatt dem Gesellschaftskörper eine Norm zu oktroyieren. Im Zentrum steht nun die Fähigkeit, Ungewissheit zu managen und mit der Bedrohung in ihrer grundlegenden Unbestimmtheit umzugehen.

(b) Die Affektpolitik folgt weniger der Linearität oder Regelmäßigkeit der Phänomene als dem, was noch nicht ist, aber jederzeit geschehen könnte oder (im Futur II) geschehen sein könnte, etwa ein erneuter exponentieller Anstieg der Infektionskurve.

(c) „In der Bedrohung als allgemeinem Bezugspunkt zeigt sich eine neue gegenwärtige Form der Biopolitik. Regieren hat nicht mehr das Einhegen von Ängsten zum Ziel, sie setzt im Gegenteil auf eine Pluralisierung der Ängste, die zu einer Transformation der Machtstrukturen führt. Wenn man das Interventionsfeld der Biopolitik als, Mixed Milieus ${ }^{6}[\ldots]$ betrachtet, als komplexe ökologische Systeme, dann zeichnet sich im affective fact ein damit korrespondierendes System der Wahrheit ab; eine Zukunftsbezogenheit der Bedrohung in ihrer konstitutiven Unbestimmbarkeit“ (Bihl 2019, 223 f.).

An die Stelle des Kondoms innerhalb der Safer-Sex-Kampagnen tritt nun die Gesichts- bzw. Mund-Nasen-Maske als Symbol der Bedrohung und der Prävention. Kondom und Maske markieren eine Grenze zwischen sich nahe kommenden potenziellen Infektionspartnern. Beide sind mit der Phantasie verbunden, sich selbst und die andere Person zu schützen. Beide nehmen ein Restrisiko in Kauf, da weder Kondom noch Maske 100\%ig schützen. Das Kondom modifiziert die sexuelle Begegnung, die Maske die verbale und mimische Kommunikation. Während das Kondom zwar öffentlich plakatiert und verkauft wird, aber doch im Raum der Intimität bleibt, hat die Maske trotz eines partiellen Vermummungs-Effekts den Vorteil, im partiellen Verhüllen Sichtbarkeit der Unterwerfung herzustellen. Sie demonstriert öffentlich: Ich schütze dich und mich in vorauseilendem Gehorsam, bevor ich durch polizeiliche Sanktionen oder kontrollierende Blicke der anderen dazu aufgefordert werde.

Machtausübung, Panoptismus, Selbst-Techniken, Subjektivierung und Kontrolle können unter Foucaults Stichwort der gouvernementalité zusammengefasst werden. Ein aktueller Literaturüberblick (Marchand et al. 2020) wählt aus 1673 zunächst gefundenen Studien 38 nach apriorisch definierten Einschlusskriterien aus und konstatiert drei Verwendungsweisen von gouvernementalité im Gesundheitswesen: eine epistemologische, eine 
Rahmen (framework) gebende sowie eine die spezifische Form von gouvernance beschreibende. Auch Jung (1950/1958) wählt eine explizit pastorale Terminologie, um die vier Stufen der psychoanalytischen Behandlung zusammenzufassen,

[...] nämlich Bekenntnis, Aufklärung, Erziehung und Verwandlung. [...] Der Uranfang aller analytischen seelischen Behandlung liegt im Vorbild des Beichtbekenntnisses. Da dieser Ursprung aber keine ursächliche Verbindung, sondern ein irrationaler, psychischer Wurzelzusammenhang ist, so ist es dem Fernstehenden nicht ohne weiteres möglich, die Grundlagen der Psychoanalyse mit der religiösen Institution der Beichte in Zusammenhang zu bringen ( $\$ \$ 122 \mathrm{f}$.).

Auch das Gespräch mit dem Patienten in der Pflegesituation hat eine „pastorale" Dimension. Allerdings erfordert der Umgang mit intimen Informationen und der Scham auf beiden Seiten (patienten- und pflegeseitig) Takt und Schutz vor Missbrauch durch Grenzverletzung (Martin 2010). Die Scham-Angst dürfte ein wesentlicher Hinderungsgrund sein, welcher dem proaktiven Ansprechen spiritueller Bedürfnisse entgegensteht (Frick 2019).

\section{Das ermöglichte und ausgeschlossene Subjekt}

Foucaults Subjekt-Begriff ist doppeldeutig (Schutijser 2019): Das Individuum wird subjektiviert (assujettissement), in dem es sich dem Wissen und der Macht unterwirft und durch sich selbst zum Subjekt wird (subjectivation). So zeigen schon seine frühen Psychiatrie-Studien, dass das Hospital ausschließt/isoliert und der Krankheit Raum, Gastfreundschaft (Hospitalität) bietet. Ähnliches gilt für die späteren Studien zur Sexualität, zur biopolitischen Sicherung des Lebens und zu den Selbst-Techniken.

Von Foucault lässt sich lernen, den Subjektbegriff nicht romantisierend oder substanzialistisch zu verwenden, sondern im Sinne einer Subjektwerdung, die je neu zu erarbeiten und ständig gefährdet ist. Diese Gefahr besteht im Ausschluss des Subjekts aus dem Diskurs, hier aus dem biomedizinischen und biopolitischen Diskurs durch äußere Kontrolle (z.B. Verbote), durch innere Kontrolle (z.B. Beschränkung der Gegenstände oder Methoden innerhalb einer Disziplin) sowie durch Verknappung und Zulassungsbeschränkung der Diskursberechtigten (Foucault 1972/1991).

Wenn das Subjekt der ursprüngliche und erste Ort ist, wo positiver Sinn erfahren, Schmerz gespürt und Entfremdung erlebt wird, und 
wenn diesen Erfahrungen eine eigene Autorität zukommt, dann verbindet sich damit das Problem einer ausgegrenzten, exkludierten Subjektivität. Was ist darunter zu verstehen? Wir werden gemeinsam nicht nur dadurch zum Subjekt des medizinisch-technischen Fortschritts, dass der entsprechende Aneignungsprozess eine bestimmte Qualität hat, sondern auch dadurch, dass wirklich alle subjektive Erfahrung für das öffentliche Bewusstsein und die öffentliche Auseinandersetzung verfügbar gemacht wird, sodass nicht einige Erfahrungsaspekte mit Fortschritt ausgeblendet werden (Schaupp 2016, 22 f.).

Die erfolgreiche Bekämpfung der Infektionskrankheiten (seit der zweiten Hälfte des 19. Jahrhunderts) wurde dadurch erkauft, dass der kranke Mensch als Subjekt aus dem medizinischen Diskurs ausgeschlossen, in ein Objekt der Medizin, genauer gesagt in den potenziellen Vektor eines Erregers, transformiert wurde. Die gegenwärtige Corona-Krise knüpft an diesen Reduktionismus an, der in der Mikrobiologie und Infektiologie methodisch legitim und notwendig ist. Problematisch werden Ausschluss und Reduktion, wenn sie Teil einer Anthropologie und einer medizinischen Ontologie werden, die in Praxis und Lehre umgesetzt wird.

Wenn der Arzt seinerseits nicht mehr als Subjekt handelt, sondern als Agent des herrschenden exkludierenden Diskurses, kommt es zu einem „doppelten Ausschluss“ des Subjektes (Lebrun 2017). Auf diesen doppelten Ausschluss der Subjektivität von Arzt und Patient bezieht sich Viktor von Weizsäckers „Einführung des Subjektes“:

Sodann hat die Einführung des Subjektes nicht etwa die Bedeutung, daß die Objektivität damit eingeschränkt würde. Es handelt sich weder um Subjektivität allein noch um Objektivität allein, sondern um die Verbindung beider. Eben darum ist nun hier doch eine Veränderung des Wissenschaftsbegriffes zu bemerken. Wissenschaft gilt nämlich hier nicht als ,objektive Erkenntnis' schlechthin, sondern Wissenschaft gilt als eine redliche Art des Umganges von Subjekten mit Objekten. Die Begegnung, der Umgang ist also zum Kernbegriff der Wissenschaft erhoben“ (Weizsäcker 1933/1997, 96).

\section{Trauer eröffnet neue Chancen für Spiritual Care}

Die durch staatliche und medizinische Institutionen angeordneten Restriktionen im Rabmen der Corona-Krise haben zablreiche persönliche und gesellschaftliche Verluste zur Folge, was Wirtschaft, Bewegungs-, Religions- und Versammlungsfreiheit usw. angeht. Dies gilt in besonderer Weise für die ganz Jungen (Lock- 
down der Kindertagesstätten, Spielplätze und Begegnungsmöglichkeiten) wie für die ganz Alten (Abschottung von Alten- und Pflegeeinrichtungen, in denen z.T. ein Gefängnis-Regime gilt), für Demente und Sterbende. Wenn diese Verluste bewusst werden, entsteht neuer Sinn für das Feblende, für die aus dem somatisierten Diskurs ausgeschlossenen Bedürfnisse.

In ausdrücklicher und definitiver Weise bezieht sich Trauer auf den Abschied von Verstorbenen und auf die Totenfürsorge vom Eintritt des Todes bis zur Bestattung sowie im Rahmen des individuellen oder kollektiven Trauerns über den Bestattungstermin hinaus - möglicherweise geknüpft an das Grab, den Ort der Bestattung.

Der Covid-19-Diskurs greift reglementierend auch in diesen Bereich des Lebens, Sterbens und Abschiednehmens ein, etwa durch Vorschriften im Umgang mit infizierten Leichen, Beschränkung der Teilnehmenden-Zahl an Bestattungsriten, Verbot von Trauergottesdiensten. Die während der Ebola-Epidemie von der WHO verwendete Formel eines „sicheren und würdevollen" Todes verknüpft bio-medizinisches und anthropologischspirituelles Denken. Im afrikanischen Kontext entstand die Rolle der „Spiritual Brokers“, die zwischen dem bio-medizinischen Modell und dem jeweiligen religiösen vermitteln (Winiger 2020). In analoger Weise lässt sich für die europäische, vergleichsweise stärker säkularisierte Situation sagen: Trauer, insbesondere Trauer um nahestehende Verstorbene, macht den Betroffenen rituelle und spirituelle Bedürfnisse sowie Ritual-Lücken, die zusätzliches Leid verursachen, bewusst. Aus dieser trauernden Bewusstwerdung kann Neues wachsen: die Erkenntnis, dass die Somatisierung des Gesundheits-Diskurses zur Bewältigung der Krise notwendig, aber eben reduktionistisch ist. Spiritual Care wird in neuer Weise "Spiritual Brokering" sein - die Verknüpfung des biomedizinischen Diskurses und Teilsystems mit der Fülle des Menschseins und die Überwindung einer reduktionistischen Medikalisierung unseres Lebens.

\section{Widerstand und Resilienz durch Spiritualität}

Spiritualität (für den späten Foucault: Selbstsorge, Zugang zur Wabrheit, freimütige Rede/parrhesia) ist eine Gestalt des subjekthaften Widerstands gegen die Macht. Dabei geht es nicht um ein romantisierendes Revoluzzertum, sondern um die Wiedergewinnung und Weiterentwicklung einer mehrdimensionalen Anthropologie für die Medizin und die Gesundheitsberufe insgesamt. Die Reduktion des Menschen auf seine virologische Rolle (Wirt, infiziert, erkrankt, immun, gestorben) ist eine Strategie, um ihn "festzulegen". Resilienz heißt, die Frage nach dem 
Menschen als dem "noch nicht festgestellten Thier" (Nietzsche) offenzuhalten: Leiblichkeit, Seele, Beziehungsfähigkeit, Spiritualität, Transzendenzverwiesenheit.

Der späte Foucault wendet sich dem Begriffsfeld Spiritualität/Sorge (souci) $\mathrm{zu}$ :

Philosophie: «Denkform, die sich danach befragt, was es dem Subjekt erlaubt, einen Zugang zur Wahrheit zu haben, [...] die versucht, die Bedingungen und Grenzen des Wahrheitszuganges des Subjekts zu bestimmen ».

Spiritualität: « die Suche, Praxis, Erfahrung, durch die das Subjekt bei sich selbst die nötigen Transformationen vornimmt, um Zugang zur Wahrheit zu erlangen» (Foucault 2001, 17).

Hatte er früher die unterwerfenden, disziplinierenden Aspekte christlicher Spiritualität und Seelenführung (im Unterschied zur sokratischen Selbstsorge: epiméleia heautûu betont, so macht er sich nun den Begriff der Spiritualität zu eigen, die er folgendermaßen charakterisiert: Die Wahrheit wird nicht durch eine eigenständige Erkenntnisanstrengung des Subjekts erlangt, sie muss dadurch "bezahlt" werden, dass das Subjekt sich bekehrt (conversion) und wandelt (transformation).

1. Eros und Askese: Das Subjekt wird aus seiner aktuellen Verfasstheit herausgerissen und emporgehoben (Eros: Liebe). Die Wandlung geschieht ferner durch Arbeit an sich selbst (Askese).

2. Durch den Zugang zur Wahrheit wird das Subjekt selbst verwandelt (effets « de retour» de la vérité sur le sujet).

8. Ausblick: Was wir durch Covid-19 gelernt haben werden

Am (ungewissen) Ende wird die Corona-Krise ein globales UngewissheitsExperiment gewesen sein. Dies schließt durchaus die Träger von Macht und Wissen ein, die mitten in unabgeschlossenen Erkenntnisprozessen Empfehlungen und Anordnungen geben, im Übrigen aber, wie alle anderen, "auf Sicht fabren". So viel ist sicher: Ungewissheit wird der Menschheit ebenso erhalten bleiben wie die Bedrohung durch Viren und andere mikrobiologische Erreger sowie eine Vielzahl pathogener Einflüsse. In einer Zeit, in der sich Institutionen „immun“ gegenüber der Spiritualität erweisen, wird Spiritual Care den Subjekten besondere Aufmerksamkeit geschenkt haben, wohl wissend, dass die neue spirituelle Kultur der Gesundheitseinrichtungen mehr ist als die Summe der Individuen. 


\section{Literatur}

Bentham, Jeremy: Panopticon, or the inspection-house : containing the idea of a new principe of construction applicable to any sort of establishment in wich persons of any description are to be kept under inspection, London: Payne 1791.

Bihl, Amelie: Biopolitik der Angst - Affekttheoretische Anschlüsse an Michel Foucault, in: Gerhards, Helene/Braun, Kathrin (Hg.): Biopolitiken - Regierungen des Lebens heute, Wiesbaden: Springer Fachmedien 2019, 209-226.

Cooper, Rosalind: Pastoral power and algorithmic governmentality, in: Theory, Culture \& Society 37 (2019) 29-52.

Foucault, Michel: Die Ordnung des Diskurses, Frankfurt a. M.: Fischer 1972/1991.

Foucault, Michel: Überwachen und Strafen. Die Geburt des Gefängnisses, Frankfurt a. M.: Suhrkamp 1975/1994.

Foucault, Michel: Omnes et singulatim: towards a criticism of political reason, The Tanner lectures on human values, Stanford: 1979/1981, 223-254.

Foucault, Michel: L'Herméneutique du sujet: Cours au Collège de France (19811982), Paris: Seuil 2001.

Frick, Eckhard: Spirituelle Anamnese, in: Roser, Traugott (Hg.): Handbuch der Krankenhausseelsorge, Göttingen: Vandenhoeck \& Ruprecht 2019, 291-300.

Frick, Eckhard: Spiritual Care in Containment-Zeiten, in: Spiritual Care 9 (2020a) [im Druck].

Frick, Eckhard: Unterwegs zum Facharzt für Spirituelle Medizin? Entwurf eines medizinisch-therapeutischen Spiritual Care-Modells zwischen Professionalisierung und Deprofessionalisierung, in: Spiritual Care 9 (2020b) [im Druck].

Frick, Eckhard/Roser, Traugott: Spiritualität und Medizin. Gemeinsame Sorge für den kranken Menschen, Stuttgart: Kohlhammer ${ }^{2} 2011$ (= Münchner Reihe Palliative Care).

Hannah, Matthew G.: Thinking Corona measures with Foucault, in: https://www.k ulturgeo.uni-bayreuth.de/de/news/2020/Thinking-Corona-measures-with-Fouca ult/index.html [14.04.2020].

Jung, Carl Gustav: Die Probleme der modernen Psychotherapie, in: Niehus-Jung, Marianne et al. (Hg.): Praxis der Psychotherapie (GW XVI), Zürich/Stuttgart: Rascher 1950/1958, 57-81.

Karle, Isolde: Perspektiven der Krankenhausseelsorge. Eine Auseinandersetzung mit dem Konzept des Spiritual Care, in: Wege zum Menschen 62 (2010) 537555 .

Lebrun, Jean-Pierre: De la maladie au malade : psychanalyse et médecine dans la cité, Toulouse: Eres 2017.

Lindemann, Gesa: Die Verschränkung von Leib und Nexistenz, in: Burow, Johannes F. et al. (Hg.): Mensch und Welt im Zeichen der Digitalisierung. Perspektiven der Philosophischen Anthropologie Plessners, Baden-Baden: Nomos 2015/2019, 47-72. 
Marchand, Jean-Sebastien/Tremblay, Dominique/Denis, Jean-Louis: Governmentality as a relevant idea for the study of healthcare networks. A scoping review, in: Nugus, Peter et al. (Hg.): Transitions and boundaries in the coordination and reform of health services. Building knowledge, strategy and leadership, Cham: Springer International Publishing 2020, 115-147.

Martin, Patrick: Pouvoir pastoral, normalisation et soins infirmiers: une analyse foucaldienne, in: Aporia 2 (2010) 25-35.

Peng-Keller, Simon: Spiritual Care: Grundgestalten, Leitmodelle und Entwicklungsperspektiven, in: Spiritual Care 9 (2020) [im Druck].

Rose, Nikolas: The politics of life itself, in: Theory, Culture and Society 18 (2001) 1-30.

Rose, Nikolas: Molecular biopolitics, somatic ethics and the spirit of biocapital, in: Social Theory \& Health 5 (2007) 3-29.

Sarasin, Philipp: Ausdünstungen, Viren, Resistenzen. Die Spur der Infektion im Werk Michel Foucaults, in: Österreichische Zeitschrift für Geschichtswissenschaften 16 (2005) 88-108.

Sarasin, Philipp: Mit Foucault die Pandemie verstehen?, in: https://geschichtederge genwart.ch/mitfoucault-die-pandemie-verstehen/ [14.04.2020].

Schaupp, Walter: Was es heißt, „Subjekt“ des medizinisch-technischen Fortschritts zu sein, in: Kröll, Wolfgang/Schaupp, Walter (Hg.): Medizin - Macht - Zwang. Wie frei sind wir angesichts des medizinischen Fortschritts?, Baden-Baden: Nomos 2016, 13-26.

Schutijser, Dennis: L'attitude moderne selon Michel Foucault: la subjectivation à la limite, in: Eidos (2019) 225-251.

Spitzer, Nils: Ungewissheitsintoleranz und die psychischen Folgen. Behandlungsleitfaden für Psychotherapie und Beratung, Berlin/Heidelberg: Springer 2019.

Weizsäcker, Viktor von: Der Gestaltkreis. Theorie der Einheit von Wahrnehmen und Bewegen, in: Janz, Dieter et al. (Hg.): Gesammelte Schriften in zehn Bänden, Bd. 4, Frankfurt a. M.: Suhrkamp 1933/1997.

Winiger, Fabian: A 'safe and dignified' death: Covid-19 and the WHO guidelines for the West African Ebola crisis, in: Spiritual Care 9 (2020) [im Druck]. 
